\title{
MOTIVACIONES DE NO FUMADORES PARA CONTINUAR CON ESTA CONDUCTA SALUDABLE
}

\author{
MOTIVATIONS OF NON-SMOKERS FOR CONTINUING \\ NOT TO SMOKE
}

\author{
VERÓNICA BEHN THEUNE ${ }^{1}$, MÓNICA CRUZ PEDREROS², \\ JULIA HUAIQUIÁN SILVA', RINA NAVEAS GONZÁLEZ ${ }^{4}$ \\ y HERNÁN SOTOMAYOR LEÓN ${ }^{5}$
}

\begin{abstract}
RESUMEN
Con el objetivo de conocer las motivaciones que apoyan el deseo de algunas personas por mantenerse sin fumar, se diseñó un estudio descriptivo transversal en una muestra de 692 adultos no fumadores pertenecientes a la Universidad de Concepción. Esta investigación permitió identificar las motivaciones intrínsecas y extrínsecas manifestadas por ellos como razones para conservar esta conducta saludable. En la recolección de los datos se utilizó la escala "Reasons for Quitting Scale" de 20 ítemes, modificada por los autores para no fumadores, y cuya confiabilidad se comprobó a través del coeficiente Alpha de Cronbach. El resultado del análisis factorial con rotación ortogonal de los 20 ítemes de la escala sugirió una solución óptima con 4 factores, considerando los ítemes que tuvieron una carga superior a 0,47. Estos cuatro factores explicaron las motivaciones por no fumar hasta un $63,11 \%$ en los funcionarios y hasta un $62,57 \%$ en los estudiantes. Los factores de mayor carga en las respuestas de los funcionarios fueron los ítemes relacionados con las categorías de refuerzo inmediato y salud, correspondientes a las motivaciones extrínsecas e intrínsecas, respectivamente. En cambio, en los estudiantes se observó mayor carga en los ítems que incluían las categorías autocontrol y presión social de las motivaciones intrínsecas y extrínsecas, respectivamente.
\end{abstract}

Palabras claves: Motivaciones, no fumador, promoción de la salud.

\begin{abstract}
In the interest of identifying the motivations that support the desire of some people to remain non-smokers, a crosssectional descriptive study of adult non-smokers was designed. This research allowed identification of intrinsic and extrinsic motivations to maintain this healthy behavior. The sample consisted of 692 non-smoker students, faculty and employees of the University of Concepcion. The instrument used for data collection was "Reasons for Quitting Scale" (Susan Curry), consisting of twenty (20) items. The researchers modified the items for non-smokers. The reliability was verified through Cronbach's Alpha Coefficient. The result of factor analysis with orthogonal rotation of the 20 items of the scale suggested an optimal solution with 4 factors, considering items that had a load higher than 0.47 . These 4 factors explained motivations to keep a non-smoking behavior of $63.11 \%$ of faculty and employees, and $62.57 \%$ of the students. The factors of greater load in the answers of faculty and employees were items related to the categories of immediate reinforcement and concern for health, corresponding to extrinsic and intrinsic motivations respectively. In contrast, factors of greater load in the student group were items that included the categories desire for self-control and social pressure corresponding to intrinsic and extrinsic motivations respectively.
\end{abstract}

Keywords: Motivation, non-smoker, health promotion.

Recepcionado: 28. 02.2003. Aceptado: 19.05.2003.

${ }^{1}$ Enfermera, Magister en Enfermería Comunitaria, Profesor Titular Depto. Enfermería Facultad de Medicina, Universidad de Concepción, Chile.

${ }^{2}$ Enfermera, Magister en Enfermería Médico Quirúrgica, Profesor Titular Depto. Enfermería, Facultad de Medicina, Universidad de Concepción, Chile.

${ }^{3}$ Enfermera, Magister en Enfermería, Profesor Asistente Depto. Enfermería, Facultad de Medicina, Universidad de Concepción, Chile.

${ }^{4}$ Licenciado en Matemáticas, Magister en Estadística, Profesor Asociado Depto. Ingeniería Matemáticas, Facultad de Ciencias Físicas y Matemáticas, Universidad de Concepción, Chile.

${ }^{5}$ Médico Cirujano, Especialista en Enfermedades Respiratorias, Profesor Titular Depto. de Medicina Interna, Facultad de Medicina, Universidad de Concepción, Chile. 


\section{INTRODUCCIÓN}

Las consecuencias del fumar para la salud están claramente documentadas por las publicaciones y los informes técnicos difundidos por la OMS/OPS (WHO 1997, 1999, 2000) y otros autores (Goic 1994; Medina, Kaempffer, Cornejo 1995; Puska, Elovainio, Vertio 1997). Es entonces fundamental iniciar el nuevo siglo con mayores éxitos en el control del tabaquismo, reconociendo que estos logros deberán ser el producto de un abordaje con perspectiva global y de aplicación de estrategias multimodales centradas tanto en el no fumador como en el fumador. El hecho de observar que el $80 \%$ de los fumadores inicia esta conducta antes de los 18 años de edad, ha movilizado alianzas de organizaciones de salud para buscar formas que permitan detener esta pandemia. Todas estas estrategias tienen como meta reducir la prevalencia de fumadores a través de dos caminos: aumentar el número de personas que se mantienen sin fumar, y aumentar el número de personas que dejan de fumar (WHO 2000).

Para el logro de la primera condición es necesario declarar el status de no fumador como el normal, promoviendo que la persona se mantenga con esta conducta saludable de por vida. Acciones en promoción de la salud proponen optimizar la salud a través de estilos de vida y ambientes saludables, que logren mejorar la calidad de vida de las personas. Las acciones de promoción de la salud y de prevención del inicio de fumar dirigidas a niños en temprana edad han demostrado ser más efectivas (Sotomayor, Behn, Rosas 1990).

Para lograr la segunda condición, la OMS recomienda dar preferencia en el apoyo y el tratamiento al fumador que manifiesta el deseo de dejar de fumar, al mismo tiempo de aumentar las barreras que dificultan el consumo, como es el aumento del precio del tabaco y normas que protejan los ambientes libres de humo de tabaco (West, McNeill, Raw 2000; Lancaster, Staed, Silagy, Sowden 2000).

Estudios realizados en nuestro país seña- lan que un 59\% de la población se mantiene sin fumar (Sotomayor, Behn, Cruz, Naveas 2000; Medina, Kaempffer, Cornejo, Hernández 1996). En Estados Unidos, sin embargo, se ha logrado aumentar la población no fumadora, con asertivas medidas de salud pública, de 57,6\% en 1965 a 75,3\% en 1997 (Eriksen, Bailey, Green, Pechacek 2000).

Para aportar conocimientos que puedan contribuir a la formulación de políticas de control del tabaquismo, los autores han estudiado las fuerzas que estimulan y apoyan a los fumadores a dejar de fumar (Behn, Sotomayor, Cruz, Naveas 2001). En el presente estudio se desea conocer las motivaciones de los no fumadores para no iniciar el consumo de cigarrillo, a pesar de la intensa presión social de los fumadores y la cada vez más audaz publicidad a favor del cigarrillo.

La literatura señala que las motivaciones han sido estudiadas y descritas por psicólogos y fisiólogos. Se definen como "el proceso de tendencia conductual del individuo de aproximación o evitación hacia un objeto para sentir satisfacción" (Todt 1991). Una de sus clasificaciones de mayor uso corresponde a las motivaciones intrínsecas y extrínsecas. Las primeras se refieren a las fuerzas que comandan las conductas de las personas desde su control interno de la salud, autoeficacia, persistencia, y las segundas se basan en el condicionamiento clásico y operante respondiendo a fuerzas externas como la recompensa, el castigo y el incentivo. La gran mayoría de los seres humanos se mueve entre las motivaciones de una completa autodeterminación (intrínsecas) hasta la completa determinación ambiental (extrínsecas), sin ser estas motivaciones excluyentes. Como entes racionales deciden cómo responder a las fuentes de motivación externas (ambientales) o internas (propias del sujeto), que constituyen el proceso mediador que orienta sus respuestas (García, Domenech 1997). Basado en lo anterior interesa identificar las motivaciones intrínsecas y extrínsecas que permiten al no fumador mantener su conducta saludable. 


\section{MATERIAL Y MÉTODO}

\section{Población}

Se realizó un estudio descriptivo de corte transversal al universo de 14.265 funcionarios y estudiantes de la Universidad de Concepción, Chile.

La muestra aleatoria estratificada de 1.418 personas se determinó con $95 \%$ de confianza y un error de muestreo $<0,025$. El status de fumador estuvo distribuido en $49 \%$ de no fumadores, $41 \%$ de fumadores y $10 \%$ de ex fumadores. Para el presente trabajo se analizaron los datos relativos a 692 no fumadores (129 funcionarios y 563 estudiantes) correspondientes al total de no fumadores de la muestra.

\section{Recolección de los datos}

Las respuestas de los participantes del estudio se obtuvieron a través de un instrumento autoadministrado que contenía consultas relativas a variables sociodemográficas y relacionadas con las motivaciones para mantenerse sin fumar. Las motivaciones se midieron con la escala "Razones por no fumar" (RNF), la cual corresponde a la escala "Reasons for Quitting Scale" (RFQ) (Curry, Wagner, Grothaus 1990), modificada por los autores para no fumadores. Sus 20 ítemes son respondidos en sistema Likert con 5 alternativas. La escala RNF identifica las motivaciones intrínsecas en sus categorías salud y autocontrol, y las motivaciones extrínsecas en sus categorías refuerzo inmediato y presión social. Su confiabilidad fue medida en una muestra piloto de 20 personas (8 funcionarios y 12 estudiantes), utilizando el coeficiente Alpha de Cronbach, que fue de 0,82 para el grupo de funcionarios y 0,80 para el grupo de estudiantes.

Los datos fueron procesados por el paquete estadístico SPSS versión 8.1, y como prueba estadística se utilizó el método multivariado de análisis factorial.

\section{RESULTADOS}

La muestra de no fumadores estudiada estuvo conformada por un $18,6 \%$ de funcionarios y un $81,4 \%$ de estudiantes. Dada la gran diferencia, tanto en lo numérico como en la edad de funcionarios y estudiantes, se consideró necesario realizar el análisis en forma separada.

El grupo de funcionarios tenía un promedio de edad de $41,3 \pm 23$ años y un $47 \%$ de hombres, mientras que el grupo de estudiantes tenía un promedio de edad de $23 \pm 6$ años $y$ un $40 \%$ de hombres.

El resultado del análisis factorial con rotación ortogonal de los 20 ítemes de la escala RNF permitió identificar el tipo de motivaciones que de preferencia presentaban los funcionarios y los estudiantes para no fumar. El análisis sugirió una solución óptima con 4 factores para ambos grupos. Sólo se analizaron los ítemes que tuvieron una carga superior a 0,47 .

Los cuatro factores identificados en el grupo de funcionarios explicaron las motivaciones por no fumar hasta un $63,11 \%$. El factor de mayor carga en sus respuestas se vio reflejado en los ítemes relacionados con la categoría de refuerzo inmediato correspondiente a las motivaciones extrínsecas, y en segundo lugar por los ítemes relacionados con la categoría salud correspondiente a las motivaciones intrínsecas. El tercer factor lo conformaron los ítemes relacionados con la presión social, y el último factor, que entregó el menor porcentaje de variabilidad, fue dado por las cargas de los ítemes de autocontrol de las motivaciones intrínsecas (Tabla 1). No existió diferencia estadísticamente significativa entre la intensidad de las motivaciones intrínsecas y extrínsecas de los funcionarios ( $\mathrm{p}$ $=0,22$ ).

Los cuatro factores identificados en el grupo de estudiantes explicaron sus motivaciones por no fumar hasta un $62,57 \%$. En este grupo se observó mayor carga en el factor que incluía ítemes de la categoría autocontrol de las motivaciones intrínsecas. El segun- 
Tabla 1. Variabilidad de los factores motivacionales en funcionarios.

\begin{tabular}{|c|c|c|c|}
\hline Fact. Motivac. & Categorías & Itemes con mayor carga & Variabl. \\
\hline I. Extrínseca. & Refuerzo inmediato & $\begin{array}{l}\text {-no deseo derrochar mi dinero } \\
\text {-no deseo contaminar el aire de mi casa } \\
\text {-no deseo que mi pelo y mi ropa huelan a cig. }\end{array}$ & 22,86 \\
\hline II. Intrínseca. & Salud & $\begin{array}{l}\text {-temo a las consecuencias en mi salud } \\
\text {-conozco gente que ha fallecido debido al fumar } \\
\text {-estoy consciente que fumar acortaría mi vida }\end{array}$ & 18,46 \\
\hline III. Extrínseca. & Presión social & $\begin{array}{l}\text {-respeto a las personas que me rodean } \\
\text {-familiares me refuerzan no fumar }\end{array}$ & 12,75 \\
\hline IV. Intrínseca. & Autocontrol & $\begin{array}{l}\text {-puedo con mi ej,. ayudar a que otros no fumen } \\
\text {-no deseo ser adicto }\end{array}$ & 9,04 \\
\hline
\end{tabular}

do factor de importancia incluía los ítemes correspondientes a presión social de las motivaciones extrínsecas, y el tercer factor estuvo representado por ítemes de la categoría salud de las motivaciones intrínsecas. El último factor respondió a la carga dada por los ítemes correspondientes al refuerzo inmedia- to; categoría que pertenece a las motivaciones extrínsecas (Tabla2). La diferencia entre las motivaciones intrínsecas y extrínsecas no fue significativa en los estudiantes $(p=0,75)$.

Al comparar los dos grupos estudiados, no hubo diferencia estadísticamente significativa en sus respuestas. Sólo se observó di-

Tabla 2. Variabilidad de los factores motivacionales en estudiantes.

\begin{tabular}{|c|c|c|c|}
\hline Fact. Motivac. & Categorías & Itemes con mayor carga & Variab. \\
\hline I. Intrínseca & Autocontrol & $\begin{array}{l}\text {-no me atrae, no lo deseo } \\
\text {-no deseo perder el control sobre mi vida }\end{array}$ & 18,35 \\
\hline II. Extrínseca & Presión social & $\begin{array}{l}\text {-me controlan para que no fume } \\
\text {-no deseo perder mis amistades que no fuman }\end{array}$ & 17,83 \\
\hline III. Intrínseca & Salud & $\begin{array}{l}\text {-temo a las consecuencias en mi salud } \\
\text {-no deseo sentirme mal }\end{array}$ & 13,73 \\
\hline IV. Extrínseca & Refuerzo inmediato & $\begin{array}{l}\text {-no deseo que mi pelo y mi ropa huelan a cig. } \\
\text {-no deseo quemar mi dinero } \\
\text {-necesito ahorrar dinero }\end{array}$ & 12,66 \\
\hline
\end{tabular}

ferencia porcentual de la variabilidad de las motivaciones expresadas por los funcionarios y por los estudiantes. En los primeros prima la motivación extrínseca, mientras que en los estudiantes predomina la motivación intrínseca (Gráfico 1). 
Gráfico 1. Variabilidad de motivaciones intrínsecas y extrínsecas de funcionarios y estudiantes de la Universidad de Concepción.

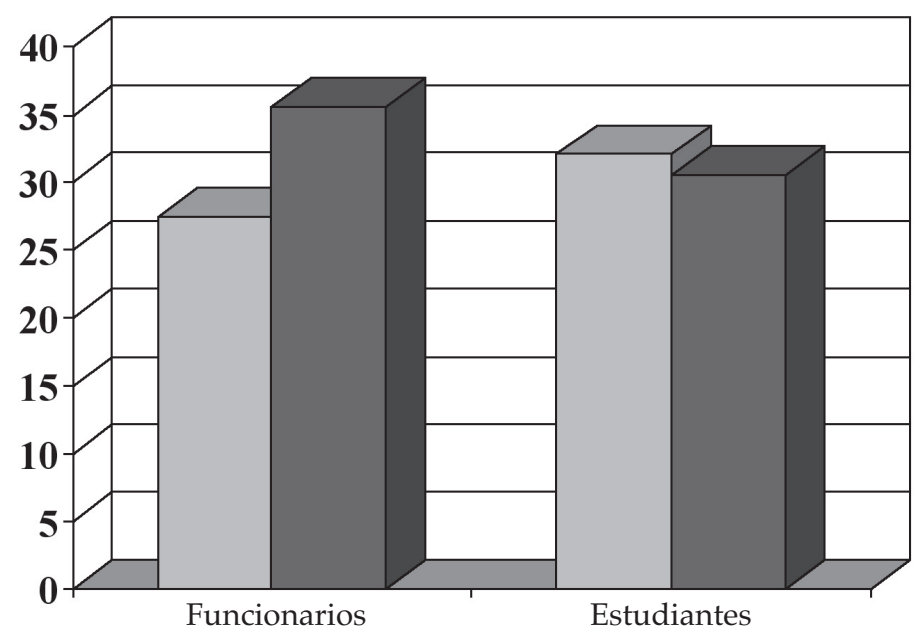

Motivación intrínseca

Motivación extrínseca

\section{DISCUSIÓN Y COMENTARIOS}

La experiencia en la aplicación de estrategias educativas para la prevención del consumo de drogas ha demostrado que no es suficiente dar a conocer a la población los peligros del consumo. Junto con señalar estos daños es igualmente importante desarrollar en las personas la capacidad para resistir las presiones negativas de los pares y la publicidad, promoviendo su autocontrol, autoestima, autoeficacia y autocuidado. Gelfer (1993) reafirma que "uno de los factores más importantes para el inicio del consumo de cigarrillos en la adolescencia es la presión de los amigos, más que de los padres, y el factor psicológico, que refleja una baja autoestima. El deseo de demostrar independencia y madurez se convierte en motivación interna para adoptar actitudes de persona adulta".

En el presente trabajo se ha estudiado el tipo de motivación que influyó en la conducta de los sujetos que se han mantenido sin fumar de por vida. El hecho de no iniciar el consumo de cigarrillo es consecuencia de una serie de factores personales y ambientales, que determinan las respuestas humanas. Interesaba identificar en los no fumadores las fuerzas que evitaron un cambio de conduc- ta, es decir, que evitaron el inicio de la conducta fumadora.

Los resultados permitieron reconocer que estas fuerzas o impulsos se manifestaron en forma diferenciada según la edad. En los jóvenes se observó que el autocontrol (que no les atrae, que no les provoca el deseo de fumar, que no desean perder el control sobre sus vidas) y la presión social (el control ejercido por otros y el no quererse diferenciar de otros) fueron las dos categorías de motivaciones de mayor carga; a diferencia de los funcionarios que señalaron la categoría de refuerzo inmediato (evitar gasto y mantener adecuadas condiciones ambientales) como primera motivación por no fumar y, en segundo lugar, su preocupación por la salud. Un resultado contrario obtuvieron Curry, Grothaus y McBride (1997) al estudiar las razones de una población de fumadores que voluntariamente manifestaban intensiones de dejar de fumar. Aquella población menor a 55 años expresaba mayor preocupación por la salud, mientras que los mayores a esa edad manifestaban preocupación por el autocontrol para dejar de fumar. Otra variante de resultados obtuvieron los autores del presente trabajo, al estudiar las motivaciones que tuvieron un grupo de ex fumadores por dejar 
de fumar. En este estudio, no hubo diferencia por edades: tanto los jóvenes como los funcionarios relacionaban su motivación principal por haber dejado de fumar con el refuerzo inmediato y por la presión social (Behn y col. 2001).

Llama la atención que los estudiantes que no fumaban tenían menor percepción del riesgo de salud y más bien determinaban su conducta por el autocontrol y la influencia social, a diferencia de los funcionarios que no fumaban, que manifestaron motivaciones económicas y de salud como las más relevantes para fundamentar su conducta no fumadora. Estas respuestas concuerdan con las características comportamentales de las personas de estos dos grupos etáreos descritas por psicólogos y sociólogos (Papalia 2001, Davidoff 2001).

En la búsqueda a través de las bases de datos electrónicas de publicaciones del área de la salud y psicología no se encontraron trabajos referidos a las características conductuales específicas de los no fumadores. Sin embargo, nos señala la literatura que las conductas, en general, que responden al estímulo de motivaciones internas o autodeterminantes se mantienen en forma duradera, porque no requieren del efecto externo de castigo, incentivo o recompensa para provocar la respuesta (Todt 1991). Esto podría explicar que las personas que no han iniciado su conducta fumadora como consecuencia de motivaciones intrínsecas, tampoco lo harán en edades posteriores. Conociendo la forma intermedia de responder de los seres humanos, entre el continuo de motivaciones internas y externas, asumimos que el no fumador responde a una conducta normal intrínsecamente motivada. Para promover esta conducta es de gran relevancia apoyar las estrategias de prevención del tabaquismo, no sólo con la información actualizada de los daños que el tabaco produce a la salud, sino también con mecanismos que incentiven el autocontrol, autoestima y autocuidado desde temprana edad.

Nuestra población chilena general se caracteriza por responder con alta sensibilidad a las exigencias o invitaciones del medio externo. Como ejemplo se puede señalar la influencia de la conducta fumadora de pares y la publicidad a favor del cigarrillo, que motiva a los fumadores en forma significativa para fumar (Medina 1996). Otro ejemplo, pero con efecto contrario, es la exitosa y masiva participación de fumadores jóvenes y mayores de nuestro país en los concursos “Déjalo y Gana" de los años 1998, 2000 y 2002 (MINSAL 2000, 2002), quienes respondieron motivados por recompensa económica, que este concurso les ofrecía. Basándonos en las situaciones anteriores es necesario reconocer el importante rol que juegan los profesionales de la salud en su responsabilidad de manifestar en forma explícita y sistemática, en cada contacto con los usuarios, la aprobación de conductas saludables, como es la de no fumar.

Esta investigación podría ampliarse en un próximo estudio considerando las variables relacionadas con el género, la religión, el nivel socioeconómico y los antecedentes de fumadores en el grupo familiar, para caracterizar en mejor forma los grupos de menor riesgo.

Se puede concluir que los resultados permiten reconocer que las motivaciones son variables importantes de considerar cuando se desea prevenir el consumo de tabaco, protegiendo al individuo expuesto al riesgo con el refuerzo de sus propias potencialidades de autocuidado y entregándole la información sistemática y actualizada sobre los daños que el tabaco produce en su salud.

\section{REFERENCIAS BIBLIOGRÁFICAS}

BEHN, V.; SOTOMAYOR, H.; CRUZ M.; NAVEAS, R. (2001). Motivaciones para dejar de fumar en funcionarios y estudiantes universitarios ex fumadores. Rev. Méd. Chile 129: 540-546.

CURRY, S.; GROTHAUS, L.; MCBRIDE, C. (1997). Reasons for quitting: intrinsic and extrinsic motivation for smoking cessation in a populationbased sample of smokers. Addict Behavior 22, (6): 727-736.

CURRY, S.; WAGNER, E.; GROTHAUS, L. (1990). Intrinsic and Extrinsic Motivation for Smoking Cessation. J Consult Clin Psychol 58 (3): 310-316. 
ERIKSEN, M; BAILEY, L.; GREEN, L.; PECHACEK, T. (2000). Tobacco Control: Development of International Public Policy. CVD Prevention 3 (2): 111-114.

GARCÍA, F.; DOMENECH, F. (1997). Motivación, aprendizaje y rendimiento escolar. Revista Electrónica de Motivación y Emoción 1.

GOIC, J. (1994). Enfermedades cardiovasculares secundarias al tabaquismo activo y pasivo. Rev Med. Chile 122: 556-562.

DAVIDOFF,L. (2001). Introducción a la psicología. (3 ed.) México. Ed. MacGraw-Hill.

LANCASTER, T.; STEAD, L.; SILAGY, C.; SOWDEN, A. (2000). Effectiveness of interventions to help people stop smoking: findings from the Cochrane Library. British Medical Journal 321, 355-358.

MEDINA, E.; KAEMPFFER, A.M.; CORNEJO, E. (1995). Tabaquismo en Santiago, 1993-1994. Revista Médica de Chile 123: 652-658.

MEDINA, E.; KAEMPFFER, A.M.; CORNEJO, E.; HERNÁNDEZ, E. (1996). Estudio de tabaquismo en siete ciudades chilenas. Cuadernos Médico Sociales 37 (2): 97-102.

OPS/OMS (2000) La epidemia de tabaquismo. Los gobiernos y los aspectos económicos del control del tabaco. Washington- USA. Publicación Científica N 577.

PAPALIA, D.; WENDKOS, S. (2001). Psicología. México. Ed. MacGraw-Hill. ISBN 968-422-194-0.

PUSKA, P.; ELOVAINIO, L; VERTIO, H. (1997). Smoke Free Europe, a Forum for Networks. Finnish Centre for Health Promotion. Ministry of Social Affairs and Health. Finland.
SOTOMAYOR, H.; BEHN, V.; ROSAS, M. (1990). Una estrategia de prevención primaria del tabaquismo en la comunidad escolar de Concepción. Anales Médicos 27 (1): 53-56.

SOTOMAYOR, H.; BEHN, V.; NAVEAS, R.; CRUZ, M. (1994). Conducta fumadora de los estudiantes ingresados a la Universidad de Concepción entre 1985 y 1990. Revista Chilena de Enfermedades Respiratorias 10: 8-15.

SOTOMAYOR, H.; BEHN, V.; CRUZ, M.; NAVEAS, R. (2000). Prevalencia del tabaquismo en académicos, no académicos y estudiantes de la Universidad de Concepción. Revista Médica de Chile 128: 977-984.

TODT, E. (1991). La motivación. Ed. Herder. Barcelona. Biblioteca Psicología 788, España.

US DEPARTMENT OF HEALTH AND HUMAN SERVICES (1990). The Health Benefits of Smoking Cessation. A Report of the Surgeon General. Rockville, MD, Centre for Disease Control, Office on Smoking and Health. (DHHS, Publication $\mathrm{N}^{\mathrm{o}}$ (CDC) 90-8416).

WEST, R.; MCNEILL, A.; RAW, M. (2000). Smoking cessation guidelines for health professionals: an update. Thorax 55: 987-999.

WHO (1997). Tobacco or Health: A global status report. Geneva. Switzterland.

WHO (1999). World Health Report. Geneva. Switzterland.

WHO (2000). European Partnership Project to reduce Tobacco Dependence. EU. Copenhagen. 